\title{
The combination of amlodipine and angiotensin receptor blocker or diuretics in high-risk hypertensive patients: rationale, design and baseline characteristics
}

\author{
W Wang ${ }^{1,3}, \mathrm{~L} \mathrm{Ma}^{3}$, Y Zhang ${ }^{1}$, Q Deng ${ }^{1}, \mathrm{M} \mathrm{Liu}^{1}$ and L Liu ${ }^{1,2}$ \\ ${ }^{1}$ Department of Evidence-Based Medicine, Cardiovascular Institute and Fuwai Hospital, Beijing, PR China \\ and ${ }^{2}$ Clinical Trial and Research Center, Beijing Hypertension League Institute, Chinese Hypertension \\ League, Beijing, PR China
}

\begin{abstract}
The Chinese Hypertension Intervention Efficacy Study (CHIEF) is a multi-centre randomized controlled clinical trial comparing the effects of amlodipine + angiotensin II receptor blocker and amlodipine +diuretics on the incidence of cardiovascular events, represented as a composite of non-fatal stroke, non-fatal myocardial infarction and cardiovascular death events in high-risk Chinese hypertensive patients. The study also evaluates
\end{abstract}

the long-term effects of lipid-lowering treatment and lifestyle modification. From October 2007 to October 2008, 13542 patients were enrolled into the study in 180 centres in China. Patients will be followed up for 4 years. There was no difference in baseline characteristics between the two blood pressure arms.

Journal of Human Hypertension (2011) 25, 271-277; doi:10.1038/jhh.2010.45; published online 6 May 2010

Keywords: antihypertensive therapy; high-risk; angiotensin II receptor blocker; diuretics; amlodipine

\section{Introduction}

Hypertension is a major cause of morbidity and mortality, and an important public health challenge worldwide. Approximately $26.4 \%$ of the adult population worldwide had hypertension in 2000, and this is expected to increase to $29.2 \%$ by $2025 .{ }^{1}$ Hypertension accounts for approximately two-thirds of all strokes and $50 \%$ of heart attacks. It causes 7.1 million premature deaths per year and $4.5 \%$ of the global burden of diseases. ${ }^{2}$ In China, according to the 2002 National Nutritional Survey, hypertension affects $18.8 \%$ of the adult population and is a leading cause of heart disease, stroke and kidney failure. ${ }^{3}$ There are more than 1.5 million new-onset strokes in China every year. ${ }^{4}$ The slope of the relationship between blood pressure (BP) and stroke in China and the Asia-Pacific region is steeper than that in western studies. ${ }^{5}$ This means that a better control of hypertension might have substantial beneficial effects on the cardiovascular morbidity

Correspondence: Dr L Ma, Department of Evidence-Based Medicine, Cardiovascular Institute and Fuwai Hospital, 167 Beilishi Road, Beijing 100037, PR China.

E-mail: maliyuan600@vip.sina.com

${ }^{3}$ These authors contributed equally to this work.

Received 27 November 2009; revised 25 March 2010; accepted 28 March 2010; published online 6 May 2010 and mortality. Many large clinical trials and metaanalyses $^{6-10}$ showed that effective BP control can significantly reduce cardiovascular morbidity and mortality. There was a $22 \%$ reduction in coronary heart disease (CHD) events and a $41 \%$ reduction in stroke for a systolic BP reduction of $10 \mathrm{~mm} \mathrm{Hg}$ or diastolic BP reduction of $5 \mathrm{~mm} \mathrm{Hg} .{ }^{10}$ Therefore, an important key to reducing the burden of hypertension-related cardiovascular disease (CVD) is to increase the proportion of patients who achieve optimal BP control.

Despite the availability of a large number of antihypertensive agents, the majority of patients with hypertension do not achieve the recommended target $\mathrm{BP}$ of $<140 / 90 \mathrm{~mm} \mathrm{Hg}$ (or $<130 / 80 \mathrm{~mm} \mathrm{Hg}$ for patients with certain comorbid conditions that increase the risk, that is, diabetes mellitus and chronic kidney disease) with antihypertensive monotherapy. The Seventh Report of the Joint National Committee on Prevention, Detection, Evaluation and Treatment of High Blood Pressure (JNC 7) ${ }^{8}$ advocates two-drug combinations using different drug classes in the majority of patients. Combination therapy with different mechanisms of actions such as a calcium channel blocker (CCB) and an angiotensin receptor blocker (ARB) or diuretics improves the overall efficacy and tolerability. CCBs are the most widely used antihypertensive drugs in 
China. The majority of large-scale clinical trials conducted in China, such as the Systolic Hypertension in China Trial (Syst-China), ${ }^{11}$ the Shanghai Trial Of Nifedipine in the Elderly (STONE) ${ }^{12}$ and the Felodipine Event Reduction (FEVER) ${ }^{13}$ chose CCBs as the basic treatment. These agents showed good efficacy in Chinese hypertensive patients, so a CCB-based regimen was chosen for this study. Diuretics, alone or in combination, are widely used as first-line hypertension treatment in China. Studies with CCB + diuretics have shown that this combination was effective in high-risk patients. ${ }^{13,14}$ Use of newer agents such as the angiotensin II receptor blocker (ARB), telmisartan, can help avoid some adverse metabolic effects, and may have additional cardiovascular protective effects. When telmisartan is used in combination with amlodipine, it can substantially offset the peripheral oedema caused by amlodipine. ${ }^{15}$ So far, no large-scale clinical trials have reported on the effects of the CCB and ARB combination on cardiovascular events.

Hypertension is often complicated with other risk factors. Cross-sectional studies have frequently reported a high prevalence of dyslipidaemia in hypertensive subjects, which considerably increases their risk of a future CHD. ${ }^{16,17}$ The recent results of the Anglo-Scandinavian Cardiac Outcomes Trial (ASCOT-LLA) $^{18}$ showed that treatment with a lipid-lowering agent, atorvastatin, in hypertensive patients reduced CHD and stroke, whereas the Antihypertensive and Lipid-Lowering Treatment to Prevent Heart Attack Trial (ALLHAT-LLT) study ${ }^{19}$ did not show a significan effect of pravastatin on either all-cause mortality or CHD.

The rationale for the CHIEF study is to try to intervene the overall risk factors of hypertension, and answer the following issues related to hypertension management: whether a newer combination of antihypertensive agents, CCB and ARB, can produce greater benefits in terms of reducing cardiovascular events than the combination of CCB and diuretics; whether lipid lowering with a statin provides additional beneficial effects in those hypertensive patients with a high-normal level of serum total cholesterol. In addition, we would like to assess the effects of lifestyle modification on the $\mathrm{BP}$ level and cardiovascular outcomes.

\section{Materials and methods}

\section{Study Design}

The CHIEF study is a large-scale, multi-centre clinical trial that involves a comparison of two treatments in a factorial design. Both treatments are in accordance with the prospective, randomized, open-labelled, blinded endpoint evaluation (PROBE) design. This trial was approved by the ethics committee. This study began in 2007, and the patients will be followed up for an average of
4 years. More than 13000 hypertension patients were enrolled from 180 clinical centres in China.

\section{Inclusion and exclusion criteria}

Antihypertensive regimen comparison. Hypertension patients aged 50-79 years were eligible if their $\mathrm{BP}$ was $140-179 / 90-109 \mathrm{~mm} \mathrm{Hg}$ at randomization after a 2-weeks run-in period. All patients had to have at least one additional cardiovascular risk factor, indicated by a history of stroke; myocardial infarction (MI); stable angina pectoris; underwent coronary artery angioplasty at least 3 months earlier; transient ischaemic attack; cardiac insufficiency (NYHA class II); peripheral vascular disease, controlled type 2 diabetes; mild or moderate chronic nephropathy (urine albumin $>300 \mathrm{mg}$ per $24 \mathrm{~h}$, or blood creatinine $>1.5 \mathrm{mg}$ per $100 \mathrm{ml}$ or $>133 \mu_{\mathrm{mol}}{ }^{-1}$ ); overweight (body mass index $>25 \mathrm{~kg} \mathrm{~m}^{-2}$ ), or obesity or abdominal obesity (waist circumference: male $\geqslant 85 \mathrm{~cm}$, female $\geqslant 80 \mathrm{~cm}$ ); abnormal blood lipid levels (total cholesterol (TC) $>5.7 \mathrm{mmol} \mathrm{l}^{-1}$, high-density lipoprotein (HDL) $<1.0 \mathrm{mmol} \mathrm{l}^{-1}$, triglycerides $>1.76 \mathrm{mmol}^{-1}$ ); family history of premature cardiovascular disease (onset before 50 years of age); age $\geqslant 65$ years; current cigarette smoker; left ventricular hypertrophy (LVH); intimal thickening or atherosclerotic plaque in the carotid arteries; hypertensive fundus oculi grade III-IV or retinal atherosclerosis grade III-IV.

BP was measured on three visits, 1 week apart, and mean values were calculated. The diagnosis of arterial hypertension was based upon elevations of either systolic ( $>140 \mathrm{~mm} \mathrm{Hg}$ ) or diastolic ( $>90 \mathrm{~mm} \mathrm{Hg}$ ) BP.

Patients with any of the following conditions were excluded from this study: secondary hypertension; history of cerebrovascular events or MI within 3 months before registration; severe cardiomyopathy or significant valvular disease; unstable angina; severe liver disease or nephropathy (alanine aminotransferase (ALT) elevation $>2 \times$ ULN or serum creatinine $>2.5 \mathrm{mg}$ per $100 \mathrm{ml}$ ); malignant tumor; gout; pregnancy or women not using contraceptives; uncontrolled diabetes (fasting plasma glucose $>10 \mathrm{mmol} \mathrm{l}^{-1}$, despite therapy); known allergies or contraindications to study drugs; considered to be unsuitable for participation in the clinical trial according to the investigator's opinion.

Lipid-lowering comparison. All subjects were eligible for the antihypertensive regimen comparison, and their serum cholesterol at screening was 4.0$6.1 \mathrm{mmol} \mathrm{l}^{-1}$. Patients were excluded if they had definite and specific indication for, or contraindication to, treatment with statin, an obvious hepatic dysfunction or unwillingness to cooperate.

Lifestyle modification. All subjects were eligible for the antihypertensive regimen comparison.

The investigators obtained written informed consent from the patients before their participation in 
the study. Investigators at the clinical sites entered the data directly into an electronic database maintained by Dorway.

\section{Run-in period}

All previous antihypertensive medication was discontinued for 2 weeks, and BP was measured at the end of the second week of the run-in period. All patients with a systolic BP (SBP) of 140-179 mm Hg and/or a diastolic BP (DBP) of 90-109 mm Hg were randomized to treatment. Patients were not enrolled if they were unable to discontinue their antihypertensive therapy for 2 weeks.

\section{Randomization}

Allocation to study treatment was carried out by internet using a central, computer-based randomization service in $2 \times 2$ factorial design. All patients were randomly allocated to amlodipine + compound amiloride (amiloride/hydrochlorothiazide; group A) or amlodipine + telmisartan (group T). Patients with serum TC levels between 4.0-6.1 $\mathrm{mmoll}^{-1}$ were further randomized to statin therapy or a standard management group. All eligible subjects were randomly assigned to an intensive lifestyle intervention group or a standard intervention group, according to the community area where the patients were resident.

\section{Study intervention}

Patients were randomized to a combination therapy with amlodipine $2.5 \mathrm{mg}$ per day and half a tablet of amiloride/hydrochlorothiazide $1.25 / 12.5 \mathrm{mg}$ (group A) or amlodipine $2.5 \mathrm{mg}$ per day and telmisartan $40 \mathrm{mg}$ per day (group T). The target BP during the treatment period was set at $<140 \mathrm{mmHg}$ systolic and $<90 \mathrm{~mm} \mathrm{Hg}$ diastolic, and $<130 \mathrm{~mm} \mathrm{Hg}$ systolic and $<80 \mathrm{mmHg}$ diastolic for diabetes or chronic kidney disease patients. If the target BP was not reached at the end of the second week after randomization, the dose of compound amiloride was titrated to one tablet per day (amiloride/ hydrochlorothiazide 2.5/25 mg) in group A, and telmisartan was titrated to $80 \mathrm{mg}$ per day in group $\mathrm{T}$. If the target BP was not reached after 4 weeks of treatment, the dosage of amlodipine in both groups was increased to $5 \mathrm{mg} /$ day (Figure 1). If the BP remained uncontrolled 2-3 months after randomization, other antihypertensive agents including ACE inhibitor, $\beta$-blockers and $\alpha$-blockers were added at the discretion of the investigator's opinion.

Patients with serum TC levels of 4.0-6.1 $\mathrm{mmol}^{-1}$ were randomized to statin intervention or standard treatment. Those in the statin treatment group receive oral simvastatin $10 \mathrm{mg}$ per day, whereas patients receiving standard treatment are managed at the discretion of their investigator's opinion. Adverse effects (for example, myalgia) are recorded and extra biochemical tests, such as ALT and creatinine kinase, should be carried out if necessary.

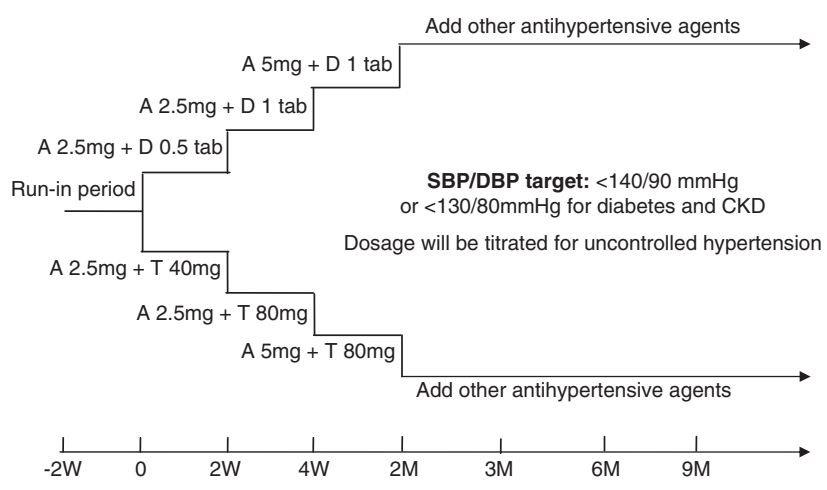

Figure 1 Study design. Abbreviations: A, amlodipine; CKD, chronic kidney disease; DBP, diastolic blood pressure; D, diuretic (amiloride $2.5 \mathrm{mg}$ and hydrochlorothiazide $25 \mathrm{mg}$ per tablet); M, month; SBP, systolic blood pressure; T, telmisartan; W, week.

Communities matched by similar socioeconomic conditions were randomly designated as an active group and a conventional group with regard to the strength of the lifestyle intervention. As required by the 2005 Chinese Hypertension Guidelines, ${ }^{20}$ patients receiving aggressive treatment were educated on a healthy lifestyle, quarterly in the first year of the study and biannually in the second year. Lifestyle changes included a reduction in body weight of $\leqslant 5 \mathrm{~kg}$ for obese patients, decreasing salt intake, $30 \mathrm{~min}$ of moderate physical activity $\geqslant 3$ times per week, reduction in cigarette smoking or smoking cessation, limiting alcohol intake or abstinence from alcohol.

\section{Follow-up and measurements}

During the screening period, patients were visited two times at 2 weeks' interval. Following randomization, each patient is reviewed at 2 weeks, 1 month, 2 months, 3 months and 3-monthly thereafter until the final visit.

At each visit BP is measured by a mercury manometer using a cuff of appropriate size. The device is calibrated every 6 months. The appearance of the first Korotkoff sound is taken as SBP, and the pressure at which the Korotkoff sound disappears (fifth phase) as DBP. On each visit, sitting BP is measured three times at 1-min intervals, after the patient has remained in a seated position for $5 \mathrm{~min}$. The average of the second and third SBP and DBP measurements is used in the analysis. At the screening and final visit, a full physical examination is carried out and blood sample is taken for risk factors and safety evaluation. Mini-mental state examination (MMSE) assessment is also conducted in the first visit, 2-year visit and last visit. The life quality and lifestyle questionnaire, and hypertension knowledge questionnaire are completed every year.

\section{Study outcomes}

The primary outcomes are the composite of nonfatal stroke, non-fatal MI and cardiovascular death. 
All suspected primary outcomes will be reviewed by an independent Endpoint Committee blinded to the study treatment. The secondary outcomes are hospitalization for heart failure, hospitalization for angina pectoris, coronary revascularization, aortic dissection, stroke, MI, cardiovascular death, allcause mortality, renal insufficiency, tumour, and new onset of atrial fibrillation and diabetes mellitus. The BP control rate, change in BP versus baseline, hypertensive fundus changes, MMSE, quality of life and lifestyle interventions are also evaluated.

\section{Sample size}

The annual incidence of cardiovascular events in elderly Chinese patients treated with CCB + diuretics was reported to be $1.52 \%$ in the FEVER study. ${ }^{13}$ If the relative difference in the incidence of cardiovascular events between the two BP arm groups is assumed to be $20 \%$, about 6000 patients are required to achieve $\alpha=0.05$ (two sided) and a power of $85 \%$. On the basis of this calculation, more than 6000 patients were randomised to each group. In patients receiving combined antihypertensive and lipid-lowering therapy, it is assumed that the incidence of composite cardiovascular events is $1.15 \%$ and the relative risk is reduced by $25 \%$.

\section{Statistical analysis}

Data will be analysed using the SAS software package. Continuous data are analysed by two-tailed unpaired $t$-test where appropriate. The $\chi^{2}$ test is used to compare categorical data. Cumulative incidence was reported by Kaplan-Meier curves for primary end points. Inter-group comparisons of cardiovascular events will include group A versus group B, statin therapy versus standard therapy, aggressive lifestyle versus conventional lifestyle interventions, a combination of antihypertensive +lipid-lowering therapy versus control therapy. The incidence of $\mathrm{LVH}$, renal dysfunction and abnormal ambulatory BP, as well as the association of LVH, renal dysfunction and ambulatory BP with the rates of cardiovascular events will also be analysed. A number of subgroup analyses will be conducted on BP, cardiovascular events and other variables. Patients' baseline characteristics, treatment compliance and adverse effects will also be analysed using descriptive statistics. Difference is considered significant at $P<0.05$.

\section{Results}

Baseline characteristics of the randomized population Patient recruitment ended in October 2008, by which time 13542 patients had been randomized to the two antihypertensive treatment regimens. Of these, 9913 patients were further randomized to lipid-lowering treatment or to the standard management group. A total of 180 clinical centres in China conducted the study. The baseline characteristics of patients in group A and group $\mathrm{T}$ are given in Table 1. No significant difference was found between the two groups.

The average age of recruits was 62 years with $49 \%$ males. The average BP at screening and randomization

Table 1 Baseline characteristics of randomized patients of the blood pressure arm (mean \pm s.d.)

\begin{tabular}{lcc}
\hline & Group A (n=6776) & Group T (n=6766) \\
\hline Sex (number (\%) men) & $3290(48.5)$ & $3286(48.6)$ \\
Age (years) & $61.5 \pm 7.7$ & $61.5 \pm 7.7$ \\
Body mass index (kg m ${ }^{-2}$ ) & $25.9 \pm 4.0$ & $26 \pm 4.0$ \\
Screening SBP (mm Hg) & $149.5 \pm 14.5$ & $149.2 \pm 14.2$ \\
Screening DBP (mm Hg) & $89.3 \pm 9.7$ & $89.5 \pm 9.6$ \\
Randomization SBP (mm Hg) & $157.3 \pm 10.8$ & $157 \pm 10.7$ \\
Randomization DBP (mm Hg) & $93.1 \pm 8.0$ & $93.2 \pm 8.0$ \\
Anti-hypertensive treatment at screening (number (\%)) & $6207(91.6)$ & $6199(91.6)$ \\
& & $1188(18.4)$ \\
Qualifying risk factors or disease & $1195(18.5)$ & $2722(41.9)$ \\
Smoking (number (\%)) & $2738(42.1)$ & $1209(19.0)$ \\
Dyslipidaemia & $1143(17.9)$ & $72(1.1)$ \\
Type 2 diabetes & $76(1.2)$ & $677(10.6)$ \\
Mild or moderate nephropathy (number (\%)) & $692(10.8)$ & $115(1.8)$ \\
History of stroke & $155(2.4)$ & $829(12.3)$ \\
History of myocardial infarction (number (\%)) & $849(12.5)$ & $102(1.6)$ \\
History of stable angina pectoris or CHD (number (\%)) & $103(1.6)$ & $120(1.9)$ \\
History of cardiac insufficiency (number (\%)) & $117(1.6)$ & $708(11.1)$ \\
History of peripheral arterial disease (number (\%)) & $668(10.4)$ & $1029(16.1)$ \\
History of transient ischaemic attack (number (\%)) & $1068(16.7)$ & \\
Family history of premature cardiovascular disease (number (\%)) & \\
\hline
\end{tabular}

Abbreviations: CHD, coronary heart disease; DBP, diastolic blood pressure; dyslipidaemia, serum cholesterol $>5.7 \mathrm{mmol} \mathrm{l}^{-1}$ or HDL-C $<1.0 \mathrm{mmoll}^{-1}$, or triglyceride $>1.76 \mathrm{mmoll}^{-1}$; group A, amlodipine+amiloride; group T: amlodipine+telmisartan; SBP, systolic blood pressure. 
was 149/89 and 157/93 mm Hg, respectively. About $92 \%$ patients were taking antihypertensive drugs before randomization. The history of stroke, history of CHD, diabetes and dyslipidaemia were 11, 12, 18 and $42 \%$, respectively, reflecting the patient inclusion criteria for CHIEF.

\section{Discussion}

CHIEF randomized in excess of 13000 patients between October 2007 and October 2008, of whom $73 \%$ were recruited into the lipid-lowering limb.

If CHIEF runs its full course, it should report at the end of 2011. CHIEF will help to define the place of the two different treatment strategies for the lowering of BP, both alone and in combination with lipid-lowering therapy and lifestyle intervention, in the prevention of cardiovascular outcomes.

The recent guidelines on hypertension ${ }^{21,22}$ and on cardiovascular prevention $^{23}$ emphasized that rather than focus on single-risk factors, focus should be on the global cardiovascular risk of an individual patient. The CHIEF study was designed to manage the hypertensive patients on the basis of an extensive intervention of the overall risk, including an initial low dose of combined antihypertensive therapy, low-dose statin-based lipid-lowering treatment and lifestyle intervention. Extensive intervention of overall risks has great potential to control hypertension and other cardiovascular risk factors, to enable more patients to reach their target BP, and further reduce the risk of cardiovascular events.

There were a number of large-scale randomized clinical trials involving combination treatment, such as ACCOMPLISH (Avoiding Cardiovascular events through COMbination therapy in Patients LIving with Systolic Hypertension), ${ }^{24,25}$ ONTARGET (Ongoing Telmisartan Alone and in combination with Ramipril Global Endpoint Trial) ${ }^{26}$ and ASCOT. ${ }^{27}$ Except the COLM study, which is ongoing in Japan and aims to compare the effects of the ARB and CCB combination with the ARB and diuretics combination on cardiovascular prevention, ${ }^{28}$ no other trials have reported the effect of the CCB and ARB combination on cardiovascular events so far.

Dyslipidaemia and hypertension commonly coexisted, ${ }^{17}$ and in the CHIEF study there were about $42 \%$ patients who had dyslipidaemia. Dyslipidaemia augmented the risk of CVD associated with hypertension. Lipid-lowering treatment in hypertensive patients could exert additional effects on the prevention of cardiovascular disease. ${ }^{16,18,29}$ The ASCOT-LLA ${ }^{29}$ has shown that treatment with atorvastatin for 3 years reduced the incidence of coronary events and stroke in hypertensive patients with a serum TC of $\leqslant 6.5 \mathrm{mmol} \mathrm{l}^{-1}$. The investigators suggested that on the basis of these findings statins should be considered in all hypertensive patients despite their cholesterol levels. ASCOT also suggested that the combination of antihypertensive and lipid-lowering therapy was better than either agent alone in terms of reducing cardiovascular events. Williams et al. ${ }^{22}$ proposed that the most effective regimen for reducing cardiovascular risk is the combination of antihypertensive and lipid-lowering therapy. In light of these findings, the determination of which combination of agents is better for improving outcomes is the focus of current clinical studies.

Treatment for high BP should be included in lifestyle modification. The recommendations for lifestyle modification were evidence-based and included adoption of a healthy dietary pattern, such as the Dietary Approaches to Stop Hypertension (DASH) diet, ${ }^{30}$ losing weight if overweight, reducing sodium intake, increasing physical activity and limiting alcohol intake. The 2005 Chinese hypertension guidelines ${ }^{20}$ also proposed the same views.

In summary, CHIEF is designed with several features, which together produce a unique trial. The CHIEF study is expected to be completed in 2011. We believe that the CHIEF study will provide useful information regarding antihypertensive therapy in elderly patients. It is hoped that the results of this study will, on completion, contribute to the evidence-based practice in the care of hypertensive patients. This study will prevent CVD, and will promote BP control rate and good adherence during long-term treatment. It is expected that the followup rate of patients on randomized treatment will be $\geqslant 90 \%$, and the $\mathrm{BP}$ control rate will be $\geqslant 70 \%$. Combination therapy is a key treatment strategy, and the management of patients with hypertension is moving towards integrated intervention. Antihypertensive treatment should not be based on BP alone; modification of lipid profile and lifestyle interventions should also be taken into account in the overall management plan.

There were two limitations of this study. First, the allocation for lifestyle management comparison was not randomized but just regionally based; there were likely to be confounders in influencing the interpretation of differences between groups. Second, the sample size calculated was mainly focused on the BP-lowering arm and lipid-lowering arm; the estimated power for lifestyle intervention maybe statistically low.

What is known about topic

- Event rates of cardiovascular disease in China differ from those in Europe and the United States. The prevalence of myocardial infarction is lower and that of stroke is higher in China. $^{4,5}$

- The above differences may be partly explained by differences in the lifestyles of China and Western countries, which are reflected in the body mass index (BMI).

What this study adds

- The present study shows that the prevalence of stroke and myocardial infarction in high-risk hypertensive patients in China is $10.7 \%$ and $2.1 \%$, respectively.

- The present study shows that the mean BMI is $26 \mathrm{~kg} \mathrm{~m}^{-2}$. 


\section{Conflict of interest}

The authors declare no conflict of interest.

\section{Acknowledgements}

The Ministry of Sciences and Technology of China has awarded a grant to assist in conducting the CHIEF study. We would like to express our gratitude to the doctors participating in the CHIEF study.

\section{References}

1 Kearney PM, Whelton M, Reynolds K, Muntner P, Whelton PK, He J. Global burden of hypertension: analysis of worldwide data. Lancet 2005; 365(9455): 217-223.

2 Bramlage P. Fixed combination of irbesartan and hydrochlorothiazide in the management of hypertension. Vascular Health and Risk Management 2009; 5: 213-224.

3 Wang L. 2002 National Nutrition and Health Survey: Report I [in Chinese], General Report. People's Medical Publishing House: Beijing, 2005.

4 Annals of Health of China Committee. 1999 Annals of Health of China [in Chinese]. People's Health Press: Beijing, China, 2000.

5 Asia Pacific Cohort Studies Collaboration. Blood pressure and cardiovascular disease in the Asia Pacific Region. J Hypertens 2003; 21: 707-716.

6 The ALLHAT Officers Coordinators for the ALLHAT Collaborative Research Group. Major outcomes in high-risk hypertensive patients randomized to angiotensin-converting enzyme inhibitor or calcium channel blocker vs diuretic: The Antihypertensive and LipidLowering treatment to prevent Heart Attack Trial (ALLHAT). JAMA 2002; 288: 2981-2997.

7 Staessen JA, Wang JG, Thijs L. Cardiovascular protection and blood pressure reduction: a meta-analysis. Lancet 2001; 358: 1305-1315.

8 Chobanian AV, Bakris GL, Black HR, Cushman WC, Green LA, Izzo Jr JL et al. Joint national committee on prevention, detection, evaluation, treatment of high blood pressure. National Heart, Lung, and Blood Institute; National High Blood Pressure Education Program Coordinating Committee. Seventh report of the Joint National Committee on Prevention, Detection, Evaluation, and Treatment of High Blood Pressure. Hypertension 2003; 42: 1206-1252.

9 Mancia G, De Backer G, Dominiczak A, Cifkova R, Fagard R, Germano G et al. Management of arterial hypertension of the European society of hypertension; European society of cardiologypar. 2007 Guidelines for the management of arterial hypertension: the task force for the management of arterial hypertension of the European society of hypertension (ESH) and of the European society of cardiology (ESC). J Hypertens 2007; 25: 1105-1187.

10 Law MR, Morris JK, Wald NJ. Use of blood pressure lowering drugs in the prevention of cardiovascular disease: meta-analysis of 147 randomised trials in the context of expectations from prospective epidemiological studies. Br Med J 2009; 338: b1665.

11 Wang JG, Staessen JA, Gong L, Liu L. Chinese trial on isolated systolic hypertension in the elderly. Systolic
Hypertension in China (Syst-China) Collaborative Group. Arch Int Med 2000; 160(2): 211-220.

12 Gong L, Zhang W, Zhu Y, Zhu J, Kong D, Pagé V et al. Shanghai trial of nifedipine in the elderly (STONE). J Hypertens 1996; 14(10): 1237-1245.

13 Liu L, Zhang Y, Liu G, Li W, Zhang X, Zanchetti A, FEVER Study Group. The Felodipine Event Reduction (FEVER) study: A randomized long-term placebo controlled trial in Chinese hypertensive patients. J Hypertens 2005; 23: 2157-2172.

14 Hashimoto J, Hirayama H, Hanasawa T, Watabe D, Asayama K, Metoki $\mathrm{H}$ et al. Efficacy of combination antihypertensive therapy with low-dose indapamide: assessment by blood pressure self-monitoring at home. Clin Exp Hypertens 2005; 27(4): 331-341.

15 Littlejohn 3rd TW, Majul CR, Olvera R, Seeber M, Kobe $\mathrm{M}$, Guthrie R et al. Study investigators. Telmisartan plus amlodipine in patients with moderate or severe hypertension: results from a subgroup analysis of a randomized, placebo-controlled, parallel-group, $4 \times 4$ factorial study. Postgrad Med 2009; 121(2): 5-14.

16 Sever P, Dahlöf B, Poulter N, Wedel H, Beevers G, Caulfield $\mathrm{M}$ et al. Potential synergy between lipidlowering and blood-pressure-lowering in the AngloScandinavian Cardiac outcomes trial. Eur Heart J 2006; 27(24): 2982-2988.

17 Kyvelou SM, Vyssoulis GP, Karpanou EA, Adamopoulos DN, Zervoudaki AI, Pietri PG et al. Effects of antihypertensive treatment with angiotensin II receptor blockers on lipid profile: an open multi-drug comparison trial. Hellenic J Cardiol 2006; 47: 21-28.

18 Sever PS, Poulter NR, Dahlof B, Wedel H, ASCOT Investigators. Antihypertensive therapy and the benefits of atorvastatin in the Anglo-Scandinavian Cardiac Outcomes Trial: lipid-lowering arm extension. J Hypertens 2009; 27(5): 947-954.

19 ALLHAT Officers and Coordinators for the ALLHAT Collaborative Research Group. The Antihypertensive and Lipid-Lowering Treatment to Prevent Heart Attack Trial. Major outcomes in moderately hypercholesterolemic, hypertensive patients randomized to pravastatin vs usual care: The Antihypertensive and Lipid-Lowering Treatment to Prevent Heart Attack Trial (ALLHAT-LLT). JAMA 2002; 288(23): 2998-3007.

20 Chinese Guidelines for Prevention Treatment of Hypertension (Revised Edition 2005). Revision Committee of Chinese Guidelines for Prevention and Treatment of Hypertension. People's Medical Publishing House, 2005.

21 Mancia G, De Backer G, Dominiczak A, Cifkova R, Fagard R, Germano G et al. 2007 Guidelines for the management of arterial hypertension: The Task Force for the Management of Arterial Hypertension of the European Society of Hypertension (ESH) and of the European Society of Cardiology (ESC). Eur Heart $J$ 2007; 28: 1462-1536.

22 Williams B, Poulter NR, Brown MJ, Davis M, McInnes GT, Potter JF et al. British Hypertension Society guidelines for hypertension management 2004 (BHSIV): summary. Br Med J 2004; 328: 634-640.

23 De Backer G, Ambrosioni E, Borch-Johnsen K, Brotons C, Cifkova R, Dallongeville J et al. European guidelines on cardiovascular disease prevention in clinical practice. Third Joint Task Force of European and other societies on cardiovascular disease prevention in clinical practice (constituted by representatives of 
eight societies and by invited experts). Eur Heart $J$ 2003; 24: 1601-1610.

24 Weber MA, Bakris GL, Dahlöf B, Pitt B, Velazquez E, Gupte $\mathrm{J}$ et al. Baseline characteristics in the Avoiding Cardiovascular events through Combination therapy in Patients Living with Systolic Hypertension (ACCOMPLISH) trial: a hypertensive population at high cardiovascular risk. Blood Pressure 2007; 16(1): 13-19.

25 Jamerson KA, Bakris GL, Wun CC, Dahlöf B, Lefkowitz M, Manfreda S et al. Rationale and design of the avoiding cardiovascular events through combination therapy in patients living with systolic hypertension (ACCOMPLISH) trial: the first randomized controlled trial to compare the clinical outcome effects of firstline combination therapies in hypertension. $A m J$ Hypertens 2004; 17(9): 793-801.

26 Teo K, Yusuf S, Sleight P, Anderson C, Mookadam F, Ramos B et al. Rationale, design, and baseline characteristics of 2 large, simple, randomized trials evaluating telmisartan, ramipril, and their combination in high-risk patients: the Ongoing Telmisartan Alone and in Combination with Ramipril Global Endpoint Trial/Telmisartan Randomized Assessment Study in ACE Intolerant Subjects with Cardiovascular Disease (ONTARGET/TRANSCEND) trials. Am Heart J 2004; 148(1): 52-61.

27 Sever PS, Dahlöf B, Poulter NR, Wedel H, Beevers G, Caulfield $\mathrm{M}$ et al. Rationale, design, methods and baseline demography of participants of the AngloScandinavian Cardiac Outcomes Trial. ASCOT investigators. J Hypertens 2001; 19(6): 1139-1147.

28 Ogihara T, Saruta T, Rakugi H, Shimamoto K, Ito S, Matsuoka $\mathrm{H}$ et al. Rationale, study design and implementation of the COLM study: the combination of OLMesartan and calcium channel blocker or diuretic in high-risk elderly hypertensive patients. Hypertens Res 2009; 32(2): 163-167.

29 Sever PS, Dahlöf B, Poulter NR, Wedel H, Beevers G, Caulfield $\mathrm{M}$ et al. Prevention of coronary and stroke events with atorvastatin in hypertensive patients who have average or lower-than-average cholesterol concentrations, in the Anglo-Scandinavian Cardiac Outcomes Trial-Lipid Lowering Arm (ASCOT-LLA): a multicentre randomised controlled trial. Drugs 2004; 64(Suppl 2): 43-60.

30 Sacks FM, Svetkey LP, Vollmer WM, Appel LJ, Bray GA, Harsha D et al. For the DASH-Sodium Collaborative Research Group: Effects on blood pressure of reduced dietary sodium the Dietary Approaches to Stop Hypertension (DASH) diet. N Engl J Med 2001; 344: 3-10.

This work is licensed under the Creative Commons Attribution-NonCommercialNo Derivative Works 3.0 Licence. To view a copy of this licence, visit http://creativecommons.org/licenses/ by-nc-nd/3.0/ 